\title{
Flexible dual-diversity wearable wireless node integrated on a dual-polarized textile patch antenna
}

\author{
Peter Vanveerdeghem, Patrick Van Torre, Christiaan Stevens, Jos Knockaert, Hendrik Rogier
}

\begin{abstract}
A new textile wearable wireless node, for operation in the $2.45 \mathrm{GHz}$ ISM band, is proposed. It consists of a dualpolarized textile patch antenna with integrated microcontroller, sensor, memory, and transceiver with receive diversity. Integrated into a garment, the flexible unit may serve for fall detection, as well as for patient or rescue-worker monitoring. Fragile and lossy interconnections are eliminated. They are replaced by very short radio-frequency signal paths in the antenna feed plane, reducing electromagnetic compatibility and signal integrity problems. The compact and flexible module combines sensing and wireless channel monitoring functionality with reliable and energy-efficient off-body wireless communication capability, by fully exploiting dual polarization diversity. By integrating a battery, a fully autonomous and flexible system is obtained. This novel textile wireless node was validated, both in flat and bent state, in the anechoic chamber, assessing the characteristics of the integrated system in free-space conditions. Moreover, its performance was verified in various real-world conditions, integrated into a firefighter garment, and used as an autonomous body-centric measurement device.
\end{abstract}

Index Terms-wireless sensors, body-centric, diversity, ISM band, textile antennas

\section{INTRODUCTION}

Smart Fabric Interactive Textile (SFIT) systems add lifesaving functionality to professional garments, as they monitor rescue workers during interventions [1], sense their environment and movements during an operation, and also establish critical communication links with other firefighters and a command post. However, further improvements, in terms of efficiency, autonomy, reliability and safety, are still needed to guarantee their market penetration and to develop the next generation of Smart Personal Protective Systems [2]. Indeed, conventional wearable body-centric wireless sensing and communication systems, for real-time tracking and monitoring of persons, rely on sensor, processor, transceiver and antenna hardware components that are often very complicated, nonflexible or bulky [3]. This makes these systems generally costly and difficult to unobtrusively integrate into garments for firefighters. Also for other applications, such as in patient and elderly monitoring [4], [5], comfort and washability may still be improved.

The indoor environments, in which these systems typically operate, suffer from severe multipath radio propagation, resulting in decreased data throughput caused by fading. In

H. Rogier, P. Vanveerdeghem and P. Van Torre are with the Information Technology Department (INTEC), Ghent University, St. Pietersnieuwstraat 41, 9000 Ghent, Belgium. E-mails: Hendrik.Rogier@UGent.be Peter.Vanveerdeghem@UGent.be Patrick.VanTorre@UGent.be

P. Van Torre is with Ghent University, IT\&C Dept., GEN research group.

P. Vanveerdeghem, C. Stevens and J. Knockaert are with Ghent University, ISP Dept., Electronics and Information Technology. addition, body shadowing plays an important role in wearable communication systems [6]. Reducing the effects of multipath fading is achieved on a single node by exploiting antenna polarization diversity [7]. The cooperation of multiple nodes provides higher-order combined polarization, spatial and pattern diversity, allowing to effectively counter fading as well as body shadowing [8]. Reliable body-centric communication requires accurate knowledge of the wireless channel characteristics in proximity of the human body [9]. This results in complex transceiver hardware that was until recently only implemented on rigid planar circuit boards and, therefore, difficult to comfortably integrate into SFIT systems.

In this article, a novel, fully-flexible, low-cost, compact, and autonomous wearable $2.45 \mathrm{GHz}$ wireless sensor node is introduced, with all its functionality fully integrated onto a dual-polarized textile patch antenna [7], [10]. The electronic circuitry, implemented on a compact, very thin, and flexible polyimide substrate, includes a microcontroller, an ADF7242 diversity transceiver, a non-volatile memory, battery and power management unit, combined with sensors. All $\mathrm{RF}$ interconnects are integrated onto the feed plane of the textile antenna, fully eliminating coaxial cables. Such a textile wireless node, realized as one compact flexible unit, enabling true non-obtrusive and comfortable integration into garments, was not documented in literature before. The efficient textile patch antenna, which serves as a platform for the active electronics, provides high antenna gain, reduces the required transmit power [11] and contributes to an energy-efficient wireless system. The textile ground plane of the wearable antenna limits the interaction with the human body, providing a wireless platform with stable characteristics and very limited radiation exposure for the user. Embedded software on the microcontroller provides wireless ad-hoc network functionality, allowing the nodes to actively forward data packets, to cooperate, share data, and synchronize over the wireless links. These properties enable the nodes to operate in a wide range of practical applications, including patient, rescue-worker or law-enforcement officer monitoring networks. The compact, autonomous, and wearable wireless node may be applied as a key building block for the construction of a modular, lowpower, body-worn system with receive diversity, by means of multiple cooperating nodes. The network topology of multiple wirelessly interconnected nodes, unobtrusively integrated in a single garment, allows us to add or remove nodes, depending on the required functionality. In addition, each node can be made washable by encapsulating the textile antenna together with the integrated electronics by a breathable Thermoplastic PolyUrethane coating (TPU) [12]. 
For research purposes, this autonomous unit can also be deployed as a channel measurement device, synchronously logging signal levels received on different autonomous nodes that are not interconnected by any wires. Conventional offbody channel measurements are generally performed with complex or expensive measurement devices [13]-[15], connected to body-worn external antennas by means of fragile coaxial cables, which hinder the test person to move freely. Truly wireless measurement nodes open new opportunities for more realistic multi-node body-centric measurement campaigns. Existing rigid wireless wearable nodes [3]-[5], used as a channel measurement device or as people-monitoring systems, are non-flexible and do not implement receive diversity.

In current literature, the number of wearable systems that integrate flexible electronic circuits with textile antennas is very limited. In [16], only a Low Noise Amplifier (LNA) is integrated, forming an active antenna that still requires a coaxial cable to be connected to transceiver units. Although in [17], the antenna and electronic system of the sensor node are integrated on the flexible substrate, the system only operates in GFSK modulation without diversity.

The article is organized as follows. Section II provides a complete description of the wearable node. In section III, the measurement results validating the performance of the system are presented, ending with the conclusions presented in section IV.

\section{WEARABLE NODE DESIGN}

\section{A. Requirements/specifications}

During rescue operations and hazardous interventions, reliable wireless communication is critical for the safe operation of firefighters. Therefore, a textile wireless node, specifically designed for comfortable integration into firefighter garments, must implement reliable and energy-efficient wireless communication. Hence, the performance of the antenna, with the system integrated onto the antenna feedplane, should not be significantly affected by the presence of the human body. The $2.45 \mathrm{GHz}$ license-exempt ISM band is selected to set up highly energy-efficient wireless links, via one of the many standards that operate in this band. Moreover, for wearable applications or for integration into a firefighter garment, the textile wireless node needs to be flexible and resistant against extreme conditions, such as high temperatures, moisture, and bending. Besides the technical specifications, the wireless node must be non-obtrusive for the user, as well as light-weight, low-cost, and energy efficient. To be useful in emergency situations, the intelligent textile system is required to be user friendly, by allowing fast and easy activation and deployment. Finally, easy replacement of certain textile nodes in the system is preferred, without the need of making wired connections between separate sub-systems.

Besides being suitable for wireless off-body communication, the wearable node should provide functionality that enables the wearable system to operate as an autonomous channel-measurement device, monitoring parameters such as Bit-Error-Rate (BER) and signal strength. For the node to be useful as a measurement device for body-centric channel characterization, sufficient memory is required to store large sets of data, obtained during the measurements. Depending on the selected modulation scheme and data rate, the node should be able to store at least a few minutes of data obtained by channel measurements. The sample period for these measurements needs to be shorter than $21.2 \mathrm{~ms}$ [18], the coherence time of the $2.45 \mathrm{GHz}$ indoor channel for a walking person. Therefore, the sampling frequency is at least $50 \mathrm{~Hz}$ on each polarization of the antenna system. Assuming 2 bytes for each data sample, providing signal strength and packetnumber information, $4 \mathrm{MB}$ of memory can store more than 5 hours of continuous measurements.

\section{B. Topology/system overview}

The key components of the newly designed wearable node are presented in the block diagram shown in Fig. 1. A top view of the circuit is given in Fig. 2. The selection of these components is motivated as follows.

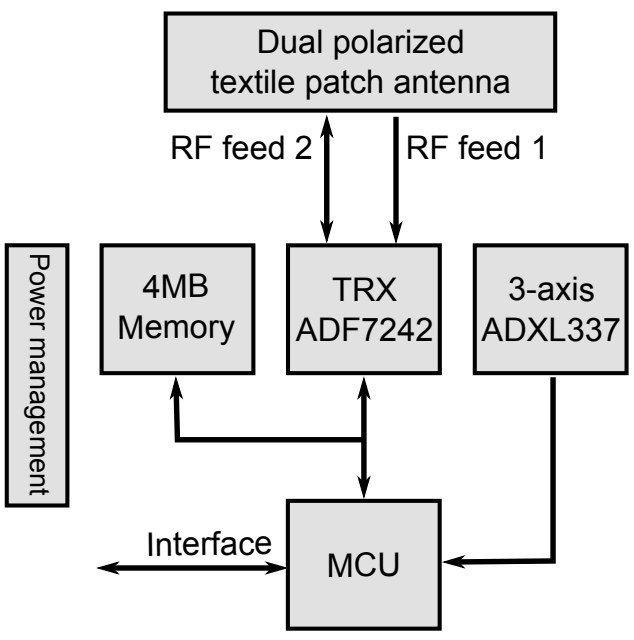

Fig. 1. Simplified block diagram of the system.

The dual-polarized textile antenna, described in detail in [7], is fully integrable into protective garments and was designed to operate in the $2.4-2.4835 \mathrm{GHz}$ ISM band, with a high (being better than $10 \mathrm{~dB}$ ) return loss, as well as excellent isolation between the antenna ports. The textile antenna, which receives two orthogonal linearly-polarized signals, is an excellent solution to reduce the undesired effect of multipath fading in wireless communications, by exploiting polarization diversity. The human body behind the ground plane of the antenna only slightly affects the performance of the antenna [7].

The heart of the transceiver system is formed by the ADF7242 integrated circuit from Analog Devices. The transceiver supports the IEEE 802.15.4-2006 2.4 GHz PHY requirements, as well as proprietary GFSK/FSK/GMSK/MSK modulation schemes. Depending on the desired application and/or data rate, one of these transmission schemes will be selected. The IEEE 802.15.4-2006 standard provides reliable data packet communication for low data-rate, wireless sensor networks. Moreover, channel measurements can also be performed with the proprietary modulation schemes, at data 
rates up to $2 \mathrm{Mbps}$, by measuring Bit-Error-Rate and Signal strength. The device features a dual-port radio-frequency (RF) interface, directly connected to the textile antenna's orthogonally polarized feeds that generate quasi-linearly polarized signals along the $\pm 45^{\circ}$ diagonals of the patch [7]. This architecture enables second-order receive polarization diversity by means of selection combining, improving the reliability of the wireless communication. A compact low-power C8051F921 microcontroller (MCU), by Silicon Laboratories, is used to process the received data and to characterize the wireless links. After processing, the signal parameters are stored in a $4 \mathrm{MB}$ flash memory, integrated into the system, providing sufficient non-volatile storage space for several hours of channel measurement data. The actions of the fire-fighter are monitored by an on-board 3-axis accelerometer. A serial port provides system access to retrieve the measurement data, or real-time data transmission to the PC at the base-station node.

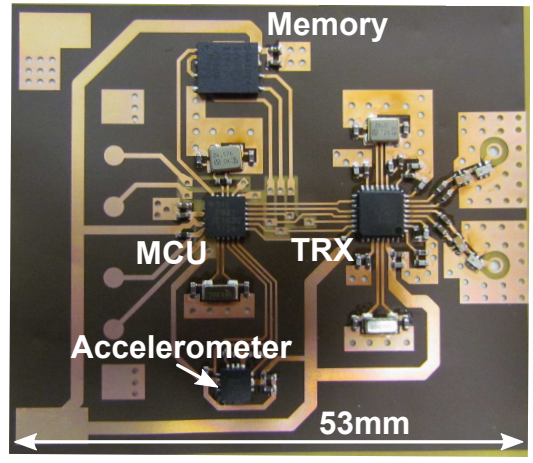

Fig. 2. Top view of the flexible wireless node circuit.

The energy-efficient, wearable node is powered by a small battery $(3.6 \mathrm{~V}, 660 \mathrm{mAh})$, ensuring the autonomy of the textile wireless node, when integrated into the garment during the course of a full rescue operation without recharging. With a power consumption lower than $90 \mathrm{~mW}$, the system can be used continuously for many hours.

\section{Material/fabrication}

The dual-polarized textile patch antenna [7], which serves as the node's platform, is constructed using FlecTron, a lowcost, conductive, electro-textile material with a thickness less than $0.25 \mathrm{~mm}$ and surface resistivity less than $0.1 \Omega / \mathrm{sq}$. This material implements the antenna patch and ground plane. Flexible, closed-cell, expanded-rubber protective foam (density $187.3 \mathrm{~kg} / \mathrm{m}^{3}$, permittivity $\epsilon_{r}=1.53$ and $\tan \delta=0.0012$ ), commonly used in protective garments for rescue workers, is applied as substrate material. The flexible foam helps to protect the electronic circuitry against external factors, such as high temperatures and humidity.

The integrated electronic system is implemented in a $9 \mu \mathrm{m}$ copper layer on a copper-on-polyimide film, UPISEL $\AA-N$ by UBE, of $25 \mu \mathrm{m}$ thickness. The production of the flexible substrate is performed by making use of an in-house photolithographic process [19] and by soldering the various components by means of reflow.

\section{Flexible circuit and antenna design}

For maximal user convenience, the flexible transceiver circuit is integrated with the textile antenna. The circuit is etched on a compact and very flexible polyimide substrate. To keep production costs low, this circuit topology is designed such that vias are avoided, as much as possible, and that only using one side is used for component placement. Thereby, the bottom layer of the circuit substrate consists almost entirely of a copper ground plane, which is connected to the back side of the antenna patch, using a conductive adhesive.

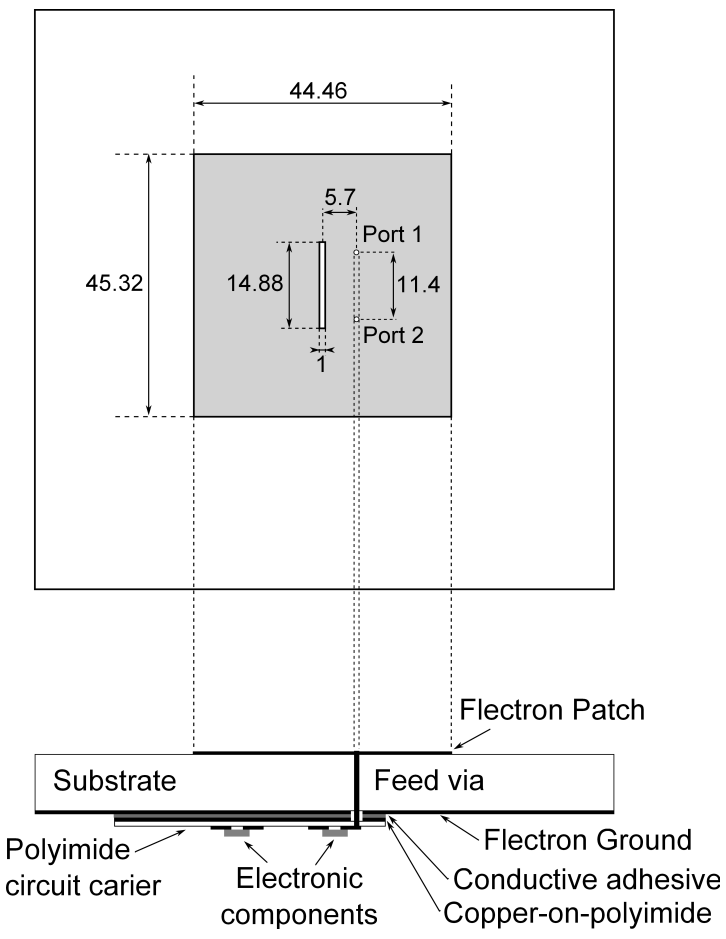

Fig. 3. Antenna cross section (dimensions in $\mathrm{mm}$ ).

The antenna is shown in Fig. 3, together with a cross section of the electronic system, integrated on the feed plane of the textile antenna. A dual-polarized antenna topology, with coaxial feeds implemented by means of vias, is adopted, since it only requires very short direct via connections between antenna patch and the transceiver's RF ports. This results in the shortest RF paths, compared to other potential feeding techniques, such as aperture coupled feeds. Moreover, the apertures in an aperture-coupled feed topology may lead to significant radiation towards the human body. Conjugate matching is applied to connect both RF output ports of the ADF7242 transceiver to Johansson Technology 2450BM14E0007 baluns, specifically designed for this chip. Each balun is followed by a $400 \mathrm{MHz}$ wide bandpass-filter (Murata LFL182G50TC1B905), to protect the input from outof-band signals. The outputs of the bandpass-filters are directly connected to the antenna feeds, ensuring the shortest possible length of the RF paths and reducing losses. The maximum output power of the transceiver, specified by Analog devices, is $+4.8 \mathrm{dBm}$. Taking into account the antenna gain and the insertion loss of the filter and balun, the maximum output power of the system is $8.9 \mathrm{dBm}$ EIRP, which complies with 
the standard (20 dBm, ETSI EN 300328 for wide band transmissions, such as in IEEE 802.15.4-2006 mode). To retain the required flexibility of the circuit, small integrated-circuit components are chosen. They are separated by a sufficiently large distance, to allow for a small bending radius. The largest rigid chip of the circuit has a footprint of $5 \times 6 \mathrm{~mm}$, thereby retaining a very flexible unit. To confirm its desired flexibility, the node is bent along a plastic tube with a curvature radius of $7.5 \mathrm{~cm}$, as shown on Fig. 4. This construction also allows the designer to perform reproducible measurements to evaluate the performance of the wireless nodes in bent condition.
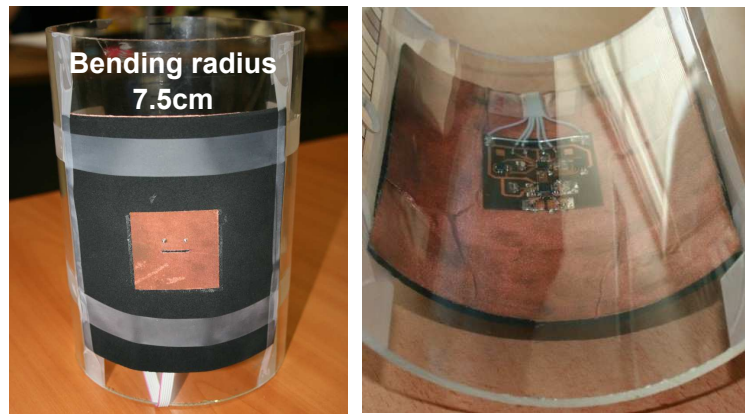

Fig. 4. The integrated system, bent around a plastic tube with a radius of $7.5 \mathrm{~cm}$.

\section{Measurements}

The indoor environment, in which the body-worn system will be deployed, exhibits multipath signal propagation. A signal transmitted along a single polarization then results in multiple reflected signals that impinge onto the receive antenna at varying polarization angles. Reception of these signals, by an antenna system with dual orthogonal polarizations, yields significant diversity gain, when combining the outputs from the two decorrelated antenna feeds. Hence, the capability of the system to receive orthogonally polarized signals in a freespace setup, leading to sufficiently decorrelated signals in a multipath environment, is an important figure of merit for the diversity performance. Therefore, the operation of the newly designed wearable node in free-space conditions is validated, focusing on the discrimination of orthogonally polarized received signals, by measuring the Cross-Polar Discrimination (XPD).

Second, the polarization of signals, transmitted or received by a wearable antenna, is sensitive to bending of the antenna and to proximity of the human body. These effects render the polarization more elliptical, instead of linear [7]. For a multipath environment, this results in higher received signal correlation and reduced diversity gain. Hence, to carefully assess the performance under different conditions, the orthogonality of the two received signals in a free-space propagation environment is tested in planar, on-body, and bent conditions.

Third, measurements are performed in an indoor scenario, with a mobile user wearing the node integrated in his firefighter jacket. These experiments evaluate the node's diversity gain realized by polarization diversity using Selection Combining (SC) or Maximum Ratio Combining (MRC). Our previous measurements [8], [10] of the body-centric channel relied on a bulky wireless testbed, placed on a cart and connected by means of coaxial cables to the antennas worn by the mobile user. During the measurement campaign, the cart with the testbed was in close proximity of the mobile user, following his/her movements. Hence, cart, testbed, and coaxial cable connections may have an impact on the wireless channel. With the proposed system, body-centric channel characterization is performed in a more realistic way. Moreover, the channel measurements will be less time consuming and more accurate, thanks to the larger data throughput, being at least 100 measurement points per second.

\section{A. Free-space propagation: orthogonality of the antenna ports}

To evaluate the orthogonality of the polarizations, the wearable node is first validated in the anechoic chamber. The measurements are performed for the stand-alone node, as well as for the node integrated into a firefighter jacket, worn by a person of size $1.85 \mathrm{~m}$ and weight $80 \mathrm{~kg}$. A transmitter, connected to a Scientific Atlanta Model 12-1.7 Standard Gain Horn antenna, continuously broadcasts data packets, which are received by the flexible node, at a distance of $4.436 \mathrm{~m}$ from the transmitter. While the horn antenna is slowly rotating at a constant rotation speed of $2 \%$, as shown in Fig. 5, the received signal power of every packet on both polarizations is logged. The variation of the received power, as a function of the polarization angle, is shown in Fig. 6. The result illustrates the complementarity of both polarizations, for both the standalone antenna and the on-body setup, in anechoic propagation conditions.
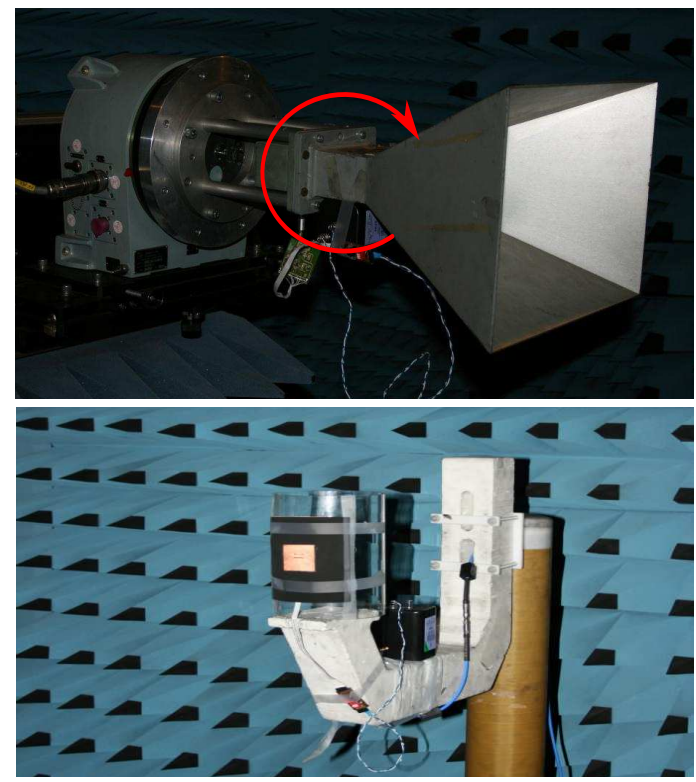

Fig. 5. Measurement setup.

From this measurement, the XPD is calculated [20], [21], defined by

$$
X P D_{Y}(d B)=10 \log _{10}\left(\frac{\left|h_{Y Y}\right|^{2}}{\left|h_{X Y}\right|^{2}}\right)
$$




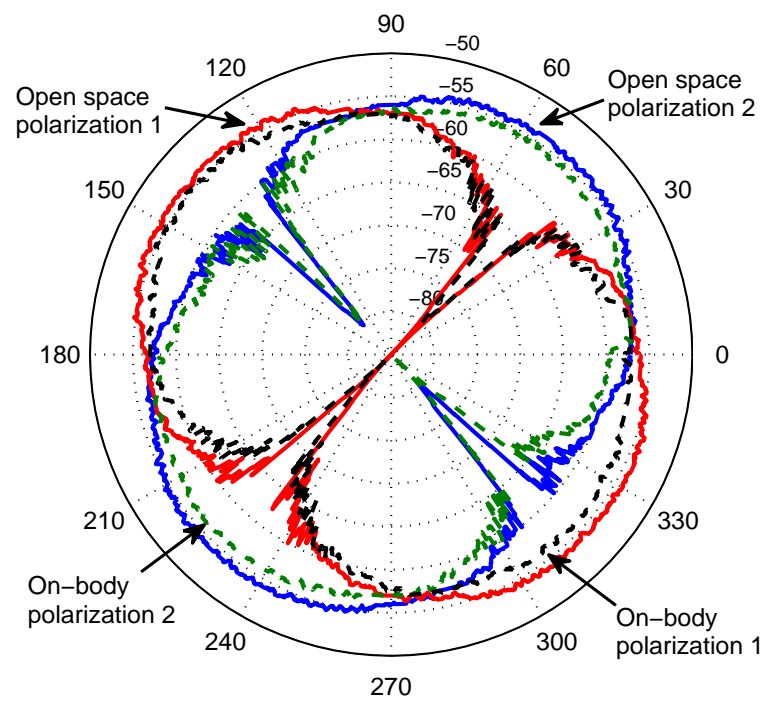

Fig. 6. Received signals power $[\mathrm{dBm}]$ on both feeds, as a function of polarization angle of the TX antenna, for the patch antenna mounted as in Fig. 3, front view, for a stand-alone node and for the node integrated into a firefighter jacket, worn by a test person.

with $\left|h_{. .}\right|^{2}$ the squared channel amplitude factor for $\mathrm{X}=+45^{\circ}$, being the co-polar polarization, and $\mathrm{Y}=-45^{\circ}$, being the crosspolar polarization. The $\mathrm{X}$ and $\mathrm{Y}$ directions are tangential to the surface of the body torso, with the zero degrees reference corresponding to a vector pointing up towards the head. The average XPD of the integrated system equals $29.1 \mathrm{~dB}$ for the stand-alone antenna and $27.1 \mathrm{~dB}$ for the on-body measurement. This confirms that the system performance is not problematically degraded by the presence of the body and that sufficiently high RF isolation is maintained between the two polarizations of the dual-polarized textile patch antenna with both terminals connected to the transceiver. These are excellent values, illustrating the suitability of the patch antenna for polarization-diversity reception. Next, the measurement was repeated with the system bent, using the setup shown in Fig. 4. A bending radius of $7.5 \mathrm{~cm}$ is realistic when deploying the textile node inside the sleeve of a garment, such that is positioned on the firefighter's arm. The variation of the received power, as a function of the radiation angle, is shown in Fig. 7. The curves now also illustrate the complementarity of both polarizations, when the wearable node is bent. Although bending slightly affects antenna impedance, port isolation, and resonance frequency [7], the average XPD is still $12.7 \mathrm{~dB}$. This guarantees satisfactory system performance in bent conditions as well.

\section{B. Multipath environment: channel measurement}

To evaluate the node's performance in a multipath environment, a fire-fighter, walking in an indoor environment with the system integrated into his fire-fighter jacket, is considered. Fig. 8 shows part of the floor plan of the building with brick walls and reinforced concrete floors at Ghent University, where the measurements were carried out. The same transmitter as in the previous measurement is located in an office at position

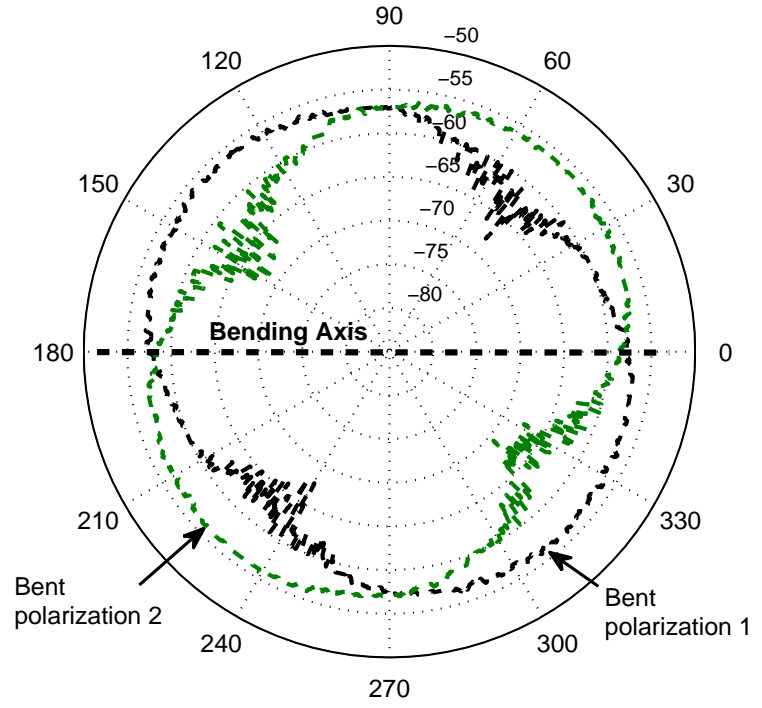

Fig. 7. Received signals power $[\mathrm{dBm}]$ on both feeds as a function of polarization angle, for the bent patch antenna mounted as in Fig. 3, front view.

TX1 in Fig. 8, while the test person, wearing the garment, walks along the path $\mathrm{A}-\mathrm{B}$ at about $1 \mathrm{~m} / \mathrm{s}$ in the neighboring offices and corridors. While data packets are continuously being transmitted, the autonomous flexible system constantly monitors the signal strength on both polarizations. As the instantaneous time-varying received power varies partially independently at both ports, 2nd-order receive diversity, by means of SC, improves the performance of the wearable node, by selecting the polarization with the strongest signal for each received data packet, as shown in Fig. 9

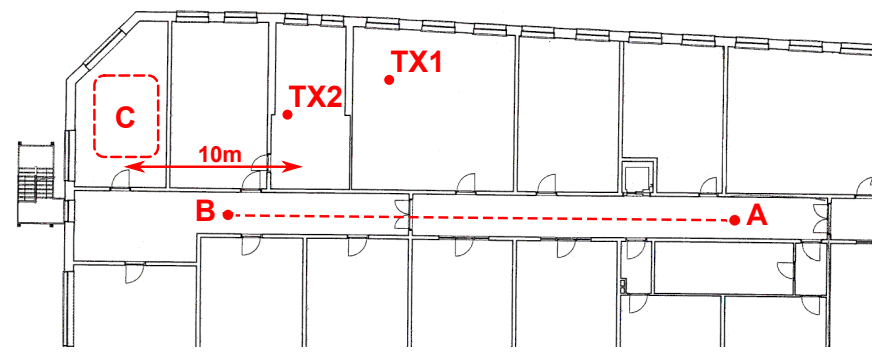

Fig. 8. Floor plan of the indoor environment, in which the channel sounding experiment was performed.

\section{Statistical analysis}

The statistical distribution of the signal levels is determined, measured within region $\mathrm{C}$ of the floor plan, shown in Fig. 8, at a distance of $10 \mathrm{~m}$ from the transmitter TX2. The analysis is performed on 32768 recorded samples per polarization, at a sample rate of $50 \mathrm{samples} / \mathrm{sec}$ on each polarization.

The graph in Fig. 10 compares the Cumulative Distribution Function (CDF) curves obtained during the measurements with and without diversity to the theoretical Rayleigh fading CDF as well as to simulated characteristics for uncorrelated Rayleigh fading channels with SC and MRC. 


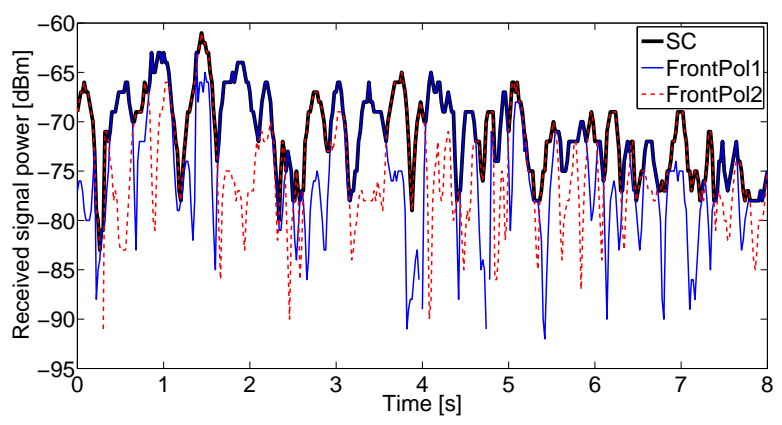

Fig. 9. Signal power received along each polarization of the antenna, when integrated into a fire-fighter jacket.

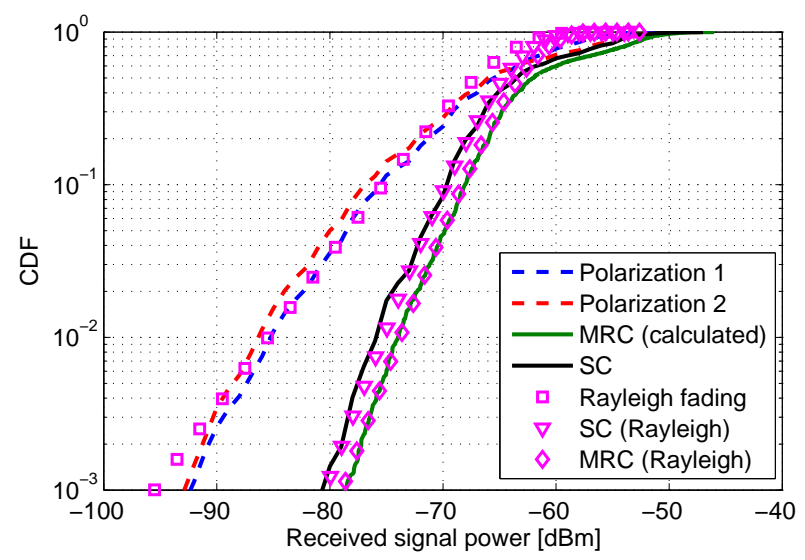

Fig. 10. Cumulative Distribution Function (CDF), for the two single polarizations, compared to the CDF for Selection Combining, Maximum Ratio Combining and characteristics for uncorrelated Rayleigh fading channels.

Only the Non-Line-of-sight (NLoS) scenario was considered here, since this scenario is the most likely to occur in the rescue worker application under study. Moreover, similar conditions were also investigated in previous measurement campaigns, serving as a reference for the current results. NLoS propagation represents a worst-case scenario, providing the most demanding conditions for the wireless off-body link.

The $10 \%$ outage probability of the dual polarized system with SC is then compared to a single polarized system. These power levels define the $10^{\text {th }}$ percentile in the CDF, meaning that the power will be higher than these values $90 \%$ of the time. SC improves the $10 \%$ outage probability by $5.5 \mathrm{~dB}$, in comparison to a single polarization. Although MRC is not implemented in this transceiver, the MRC gain is calculated and drawn in Fig. 10. MRC increases the $10 \%$ outage probability by $7.5 \mathrm{~dB}$, compared to a single polarization. Considering the $1 \%$ outage probability, SC and MRC produce a gain of $9.5 \mathrm{~dB}$ and $11.5 \mathrm{~dB}$, respectively, in comparison to a single polarization. The observed deviation from the Rayleigh characteristics below the $1 \%$ level is due to the limited number of recorded signals.

\section{Power consumption}

The power consumption is highly dependent on the operating mode of the node. In sleep mode, the total power consumption is less than $10 \mu \mathrm{W}$. The measured power consumption for the wearable system, in full operation, equals $90 \mathrm{~mW}$ $(27 \mathrm{~mA}, 3.3 \mathrm{~V})$. Hence, even in continuous full operation, the system can be used during many hours, without recharging the battery. For the experiments, a low drop voltage regulator is mounted on top of the battery, causing an additional power dissipation of approximately $9 \mathrm{~mW}$. In addition, at the power input connector, an inversely polarized diode is placed for polarity protection.

\section{CONCLUSIONS}

The first autonomous textile wireless node, fully integrated onto a flexible dual-polarized textile patch antenna, was presented. This design is particularly suited to perform bodycentric channel measurements with all the equipment integrated into clothing. The wearable node enables 2 nd-order receive diversity, by means of selection combining. Thanks to the integration of all the circuit components on the antenna feed plane, directly below the antenna patch and the ground plane, fragile and lossy interconnections are avoided. Moreover, the small size of the wearable node eliminates electromagnetic compatibility and signal integrity issues. This ultra-flexible and compact wireless transceiver/sensor node can be unobtrusively integrated into rescue worker garments, making it useful for a plethora of wearable applications. The flexible node exhibits a modular topology and is easily expandable to create a customizable textile module. Therefore, this new low-cost system is a step forward in designing comfortable commercial firefighter protective systems.

\section{ACKNOWLEDGEMENTS}

This research was partially funded by the Inter-University Attraction Poles Program initiated by the Belgian Science Policy Office.

\section{REFERENCES}

[1] G. Magenes, D. Curone, L. Caldani, and E.L. Secco, "Fire fighters and rescuers monitoring through wearable sensors: The proetex project", in Engineering in Medicine and Biology Society (EMBC), 2010 Annual International Conference of the IEEE, 2010, pp. 3594-3597.

[2] IWT Belgium, the Flemish government agency for Innovation by Science and Technology, "Smart@fire, a groundbreaking european project (FP7) to encourage companies and researchers and provide them with financial means to develop innovative ICT solutions that better protect firefighters and help prevent accidents, and to integrate them into smart personal protective equipment (PPE).", June 2013.

[3] M.O. Munoz, R. Foster, and Yang Hao, "On-body channel measurement using wireless sensors", Antennas and Propagation, IEEE Transactions on, vol. 60, no. 7, pp. 3397-3406, 2012.

[4] Lingfei Mo, Shaopeng Liu, R.X. Gao, D. John, J.W. Staudenmayer, and P.S. Freedson, "Wireless design of a multisensor system for physical activity monitoring", Biomedical Engineering, IEEE Transactions on, vol. 59, no. 11, pp. 3230-3237, 2012.

[5] Yao-Chiang Kan and Chun-Kai Chen, "A wearable inertial sensor node for body motion analysis", Sensors Journal, IEEE, vol. 12, no. 3, pp. 651-657, 2012.

[6] I. Khan, I. Ullah, and P.S. Hall, "Transmit-receive diversity for 2 x 2 multiple-input multiple-output channel in body area networks", Microwaves, Antennas Propagation, IET, vol. 5, no. 13, pp. 1589-1593, 2011.

[7] L. Vallozzi, H. Rogier, and C. Hertleer, "Dual Polarized Textile Patch Antenna for Integration into Protective Garments", IEEE Antennas Wireless Prop. Lett., vol. 7, pp. 440-443, 2008. 
[8] Patrick Van Torre, Luigi Vallozzi, Lennert Jacobs, Hendrik Rogier, Marc Moeneclaey, and Jo Verhaevert, "Characterization of measured indoor off-body MIMO channels with correlated fading, correlated shadowing and constant path loss", Wireless Communications, IEEE Transactions on, vol. 11, no. 2, pp. 712-721, 2012.

[9] P. S. Hall and Y. Hao, "Antennas and propagation for body centric communications", in Antennas and Propagation, 2006. EuCAP 2006. First European Conference on, 2006, pp. 1-7.

[10] L. Vallozzi, P. Van Torre, C. Hertleer, H. Rogier, M. Moeneclaey, and J. Verhaevert, "Wireless communication for firefighters using dualpolarized textile antennas integrated in their garment", Antennas and Propagation, IEEE Transactions on, vol. 58, no. 4, pp. $1357-1368$, April 2010.

[11] P. Vanveerdeghem, B. Jooris, P. Becue, P. Van Torre, H. Rogier, I. Moerman, and J. Knockaert, "Reducing power consumption in body-centric ZigBee communication links by means of wearable textile antennas", in 2nd International Workshop on Measurement-based Experimental Research, Methodology and Tools, 2013.

[12] Maria Lucia Scarpello, Ilda Kazani, Carla Hertleer, Hendrik Rogier, and Dries Vande Ginste, "Stability and efficiency of screen-printed wearable and washable antennas", Antennas and Wireless Propagation Letters, IEEE, vol. 11, pp. 838-841, 2012.

[13] A. Alomainy, A. Sani, A. Rahman, J.G. Santas, and Yang Hao, "Transient characteristics of wearable antennas and radio propagation channels for ultrawideband body-centric wireless communications", Antennas and Propagation, IEEE Transactions on, vol. 57, no. 4, pp. 875-884, 2009.

[14] P.A. Catherwood and W.G. Scanlon, "Body-centric ultra-wideband multi-channel characterisation and spatial diversity in the indoor environment", Microwaves, Antennas Propagation, IET, vol. 7, no. 1, pp. 61-70, 2013.

[15] Q.H. Abbasi, A. Sani, A. Alomainy, and Yang Hao, "On-body radio channel characterization and system-level modeling for multiband OFDM ultra-wideband body-centric wireless network", Microwave Theory and Techniques, IEEE Transactions on, vol. 58, no. 12, pp. 34853492, 2010.

[16] Frederick Declercq and Hendrik Rogier, "Active integrated wearable textile antenna with optimized noise characteristics", Antennas and Propagation, IEEE Transactions on, vol. 58, no. 9, pp. 3050-3054, 2010.

[17] B. Huyghe, J. Vanfleteren, and J. Doutreloigne, "Design of flexible, low-power and wireless sensor nodes for human posture tracking aiding epileptic seizure detection", in Sensors, 2009 IEEE, 2009, pp. 19631966.

[18] H. MacLeod, C. Loadman, and Z. Chen, "Experimental studies of the 2.4-GHz ISM wireless indoor channel”, in Communication Networks and Services Research Conference, 2005. Proceedings of the 3rd Annual, 2005, pp. 63-68.

[19] Jonathan Govaerts, Wim Christiaens, Erwin Bosman, and Jan Vanfleteren, "Fabrication processes for embedding thin chips in flat flexible substrates", Advanced Packaging, IEEE Transactions on, vol. 32, no. 1, pp. 77-83, 2009.

[20] F. Quitin, C. Oestges, F. Horlin, and P. De Doncker, "Polarization measurements and modeling in indoor NLOS environments", Wireless Communications, IEEE Transactions on, vol. 9, no. 1, pp. 21-25, 2010.

[21] L. Akhoondzadeh-asl, P.S. Hall, Y. Nechayev, and I. Khan, "Depolarization in on-body communication channels at $2.45 \mathrm{GHz}$ ", Antennas and Propagation, IEEE Transactions on, vol. 61, no. 2, pp. 882-889, 2013. 